\title{
Strongly determined types and G-compactness
}

\author{
by
}

\section{A. A. Ivanov (Wrocław)}

\begin{abstract}
We study connections between G-compactness and existence of strongly determined types.
\end{abstract}

0. Introduction. Theories admitting strongly determined types were introduced in [6]. It was shown there that the class of these theories is very wide. In particular it contains all stable theories, PA, the theory of the field $\mathbb{Q}_{p}$, all o-minimal theories and theories of various finitely homogeneous structures. Some other interesting examples can be found in Section 2 of [6]. A great deal of effort of [6] was concentrated on demonstration that many results from stability theory can be carried over to the much more general context of theories admitting strongly determined types.

Hrushovski has pointed out to the authors of [6] that a correct version of Lemma 2.2 of [6] must involve G-compactness. This has become the starting point of this paper. Here we study connections between existence of strongly determined types and G-compactness. In particular we repair Lemma 2.2 of [6] and several applications of this lemma from [6]. In Section 1.1 we give some necessary information about strongly determined types. In Section 1.2 we introduce G-trivial strongly determined types and in Section 2 we use them in the main result of the paper asserting that admitting strongly determined 1-types and type-definability of the equivalence relation of being of the same Lascar strong type imply that this equivalence relation coincides with the relation of being of the same strong type. In particular we have this conclusion if the theory is simple and admits strongly determined 1-types. Some further questions arising in the case of simple theories are discussed in Section 3. We give some examples there.

It turns out that the scheme of the definition of strongly determined types can be applied in the case of bounded equivalence relations. This leads us

2000 Mathematics Subject Classification: Primary 03C45.

Key words and phrases: G-compactness, strongly determined type. 
to KP-determined and L-determined types, very close notions which become more natural in some situations. We discuss this in Section 4.

This research was supported by KBN grant 2 P03A 007 19, and basically carried out when the author held a visiting position at the Institute of Mathematics, Polish Academy of Sciences. The author is grateful to the referee for helpful remarks.

In this paper, $T$ will denote a first-order theory over a countable language. The symbols $M, N$ will denote models of $T$, which are assumed to be elementary substructures of a sufficiently saturated monster model $\mathbf{C}$. We use $A, B$ to denote subsets of $\mathbf{C}$, assumed to be much smaller than $\mathbf{C}$. If $\bar{a}$ is a tuple, we often abuse notation by writing $\bar{a} \in M$. If no such restriction is given, then $\bar{a}$ is assumed just to live in C. If $r(\bar{x})$ is a type in (possibly infinitely many) variables $\bar{x}$, we denote by $r(M)$ the set of tuples (sequences) from $M$ which realize $r$. For any structure $M$ and $A \subseteq \mathbf{C}$, define $\operatorname{Aut}(M / A)$ to be the group of automorphisms of $M$ which are partial maps $\mathbf{C} \rightarrow \mathbf{C}$ fixing $A$ pointwise.

The following definitions and facts are partially taken from [10]. An $A$ hyperimaginary is an equivalence class of an $A$-type-definable equivalence relation [10]. It becomes an imaginary if the equivalence relation is $A$ definable. Let $\operatorname{bdd}(A)$ be the set of all $A$-hyperimaginaries which have a small orbit under $\operatorname{Aut}(\mathbf{C} / A)$. A hyperimaginary $e$ is bounded if $e \in \operatorname{bdd}(\emptyset)$. In this paper we usually consider hyperimaginaries depending on finitely many variables.

We recall that for $\bar{c} \in \mathbf{C}$ the strong type (resp. KP-strong type) of $\bar{c}$ over $A$ is just $\operatorname{tp}\left(\bar{c} / \operatorname{acl}^{\mathrm{eq}}(A)\right)(\operatorname{resp} \cdot \operatorname{tp}(\bar{c} / \operatorname{bdd}(A)))$. We write $S\left(\operatorname{acl} l^{\mathrm{eq}}(A)\right)$ (resp. $S(\operatorname{bdd}(A)))$ for the set of strong (resp. KP-strong) types over $A$ depending on variables of the sorts of the language of $T$. It is worth noting here that a straightforward definition allows us to consider types of hyperimaginaries over hyperimaginaries. We may also define $\operatorname{acl}^{\mathrm{eq}}(\mathcal{A})(\operatorname{resp} \operatorname{bdd}(\mathcal{A}))$, for $\mathcal{A}$ consisting of hyperimaginaries, as $\mathcal{A}$ together with the set of all equivalence classes of $\mathcal{A}$-definable ${ }^{1}$ ) (resp. $\mathcal{A}$-type-definable) equivalence relations having finite (resp. small) orbits under $\operatorname{Aut}(\mathbf{C} / \mathcal{A})$. On the other hand, in this paper we concentrate on theories admitting strongly determined types and the corresponding definition (see [6]) involves types over sets of basic sorts. Therefore in all our statements sets of hyperimaginaries will appear in some particular situations when all imaginaries (hyperimaginaries) from $\mathcal{A}$ belong to $\operatorname{acl}^{\text {eq }}(\mathcal{A} \cap \mathbf{C})(\operatorname{resp}$. $\operatorname{bdd}(\mathcal{A} \cap \mathbf{C}))$; here by $\mathcal{A} \cap \mathbf{C}$ we denote the part of $\mathcal{A}$ of the sorts of the language of $T$. Then $\operatorname{acl}^{\mathrm{eq}}(\mathcal{A})($ resp. $\operatorname{bdd}(\mathcal{A}))$ is just $\operatorname{acl}^{\mathrm{eq}}(\mathcal{A} \cap \mathbf{C})($ resp. $\operatorname{bdd}(\mathcal{A} \cap \mathbf{C}))$.

$\left({ }^{1}\right)$ By an $\operatorname{Aut}(\mathbf{C} / \mathcal{A})$-invariant formula with parameters from $\mathbf{C}$. 
For $\delta \in\{1,2, \ldots, \omega\}$ and a set $A$ let $E_{\mathrm{L}}^{A, \delta}$ be the finest bounded $\operatorname{Aut}(\mathbf{C} / A)$ invariant equivalence relation on $\delta$-sequences. The classes of $E_{\mathrm{L}}^{A, \delta}$ are called Lascar strong types $\left({ }^{2}\right)$. For $A \subset \mathbf{C}$ the relation $E_{\mathrm{L}}^{A, \delta}$ can be characterized as follows $([1]):(\bar{a}, \bar{b}) \in E_{\mathrm{L}}^{A, \delta}$ if there are models $M_{1}, \ldots, M_{n}<\mathbf{C}$ containing $A$ and sequences $\bar{a}_{0}(=\bar{a}), \ldots, \bar{a}_{n}(=\bar{b})$ such that $\operatorname{tp}\left(\bar{a}_{i} / M_{i+1}\right)=$ $\operatorname{tp}\left(\bar{a}_{i+1} / M_{i+1}\right), 0 \leq i<n$. Equivalently $(\bar{a}, \bar{b}) \in E_{\mathrm{L}}^{A, \delta}$ if there are sequences $\bar{a}_{0}(=\bar{a}), \ldots, \bar{a}_{n}(=\bar{b})$ such that each pair $\bar{a}_{i}, \bar{a}_{i+1}, 0 \leq i<n$, extends to an infinite indiscernible sequence over $A$ ([1]).

Let $A \subset \mathbf{C}$. We denote by $E_{\mathrm{KP}}^{A, \delta}$ the finest bounded $A$-type-definable equivalence relation on $\delta$-sequences, and by $E_{\mathrm{Sh}}^{A, \delta}$ the intersection of all finite $A$-definable equivalence relations on $\delta$-sequences. Sequences $\bar{a}$ and $\bar{b}$ have the same strong (resp. KP-strong) types over $A$ if and only if they are $E_{\mathrm{Sh}}^{A, \delta}$ equivalent (resp. $E_{\mathrm{KP}}^{A, \delta}$-equivalent). It is known from [9] that for $\omega$-categorical theories and for finite $A \subset \mathbf{C}$ and $\delta, E_{\mathrm{Sh}}^{A, \delta}=E_{\mathrm{KP}}^{A, \delta}=E_{\mathrm{L}}^{A, \delta}$. For small theories $E_{\mathrm{Sh}}^{A, \delta}=E_{\mathrm{KP}}^{A, \delta}([7],[8],[12])$.

In the paragraphs above we have slightly changed the notation from [1]. The reason is that in the case when $X$ is a set defined over $A$ and $B$, the notation $E_{\mathrm{KP}}^{X}$ can be interpreted as both $E_{\mathrm{KP}}^{A, n}$ and $E_{\mathrm{KP}}^{B, n}$. These relations are not necessarily the same. Below we often use just $E_{\mathrm{KP}}$ when it is clear over which set we work.

For any structure $M$ and $A \subseteq \mathbf{C}$, define $\operatorname{Aut}_{\mathrm{KP}}(M / A):=\operatorname{Aut}(M / \operatorname{bdd}(A))$ and $\operatorname{Aut}_{\mathrm{Sh}}(M / A):=\operatorname{Aut}\left(M / \mathrm{acl}^{\mathrm{eq}}(A)\right)$. We call the elements of $\operatorname{Aut}_{\mathrm{Sh}}(M / A)$ (resp. $\left.\operatorname{Aut}_{\mathrm{KP}}(M / A)\right)$ Shelah strong automorphisms over $A$ (resp. KP-strong automorphisms over $A)$; the elements of $\operatorname{Aut}_{\mathrm{Sh}}(M)=\operatorname{Aut}_{\mathrm{Sh}}(M / \emptyset)$ are called Shelah strong automorphisms $\left({ }^{3}\right)$. We talk similarly of Shelah strong (resp. KP-strong) elementary maps over $A$.

Let $M$ be a saturated structure of uncountable cardinality and let $\operatorname{Aut}_{\mathrm{L}}(M)$ be the group of all Lascar strong automorphisms (fixing the classes of all bounded invariant equivalence relations). Then $\operatorname{Gal}_{\mathrm{L}}(\mathrm{Th}(M))=$ $\operatorname{Aut}(M) / \operatorname{Aut}_{\mathrm{L}}(M)$, the Galois group of $\operatorname{Th}(M)$, does not depend on $M$. The following group extensions show relationships among these notions:

$$
1 \rightarrow \operatorname{Aut}_{\mathrm{KP}}(M) / \operatorname{Aut}_{\mathrm{L}}(M) \rightarrow \operatorname{Gal}_{\mathrm{L}} \rightarrow \operatorname{Gal}_{\mathrm{KP}}=\operatorname{Aut}(M) / \operatorname{Aut} \mathrm{KP}(M) \rightarrow 1
$$

and

$$
1 \rightarrow \operatorname{Aut}_{\mathrm{Sh}}(M) / \operatorname{Aut}_{\mathrm{L}}(M) \rightarrow \mathrm{Gal}_{\mathrm{L}} \rightarrow \operatorname{Gal}_{\mathrm{Sh}}=\operatorname{Aut}(M) / \operatorname{Aut}_{\mathrm{Sh}}(M) \rightarrow 1 .
$$

It is known that the groups $\mathrm{Gal}_{\mathrm{KP}}$ and $\mathrm{Gal}_{\mathrm{Sh}}$ are compact. The theory

\footnotetext{
$\left({ }^{2}\right)$ Usual strong types can be called Shelah strong types.

$\left({ }^{3}\right)$ They were introduced by Lascar as strong automorphisms.
} 
$\operatorname{Th}(M)$ is called $G$-compact if $\mathrm{Gal}_{\mathrm{L}}=\mathrm{Gal}_{\mathrm{KP}}$. The main results of the paper are connected with the question when $\mathrm{Gal}_{\mathrm{L}}=\mathrm{Gal}_{\mathrm{Sh}}$.

1. Strongly determined types and equivalence relations. We start this section with a short introduction to strongly determined types [6].

1.1. Strongly determined types. The following definition is taken from [6]. Let $A \subset \mathbf{C}$ and $\mathcal{A} \subset \operatorname{acl}^{\mathrm{eq}}(A)$ with $A \subseteq \mathcal{A}$. If $q(\bar{y}) \in S\left(\operatorname{acl}^{\mathrm{eq}}(A)\right)$ we say that a type $p(\bar{x}, \bar{y}) \in S(\mathcal{A})$ is a $q$-consistent $\bar{x}$-type if for any sequence $\bar{a}_{1}, \ldots, \bar{a}_{n}$ of realizations of $q$ the set $\bigcup\left\{p\left(\bar{x}, \bar{a}_{i}\right): 1 \leq i \leq n\right\}$ is consistent. A strongly determined type over $\mathcal{A}$ is a function $\varrho$ which assigns a $q$-consistent $\bar{x}$-type $\varrho(q)(\bar{x}, \bar{y})$ to every $q(\bar{y}) \in S\left(\operatorname{acl}^{\mathrm{eq}}(A)\right)$, and is monotonic: if $\bar{y}^{\prime}$ is a subtuple of $\bar{y}$ and $q(\bar{y}), q^{\prime}\left(\bar{y}^{\prime}\right) \in S\left(\mathrm{acl}^{\mathrm{eq}}(A)\right)$ with $q(\bar{y}) \vdash q^{\prime}\left(\bar{y}^{\prime}\right)$, then the restriction of $\varrho(q)(\bar{x}, \bar{y})$ to $\bar{x} \bar{y}^{\prime}$ is $\varrho\left(q^{\prime}\right)\left(\bar{x}, \bar{y}^{\prime}\right)$.

Suppose that $\varrho$ is a strongly determined type over $\mathcal{A}$. For every $B \subset \mathbf{C}$ define

$$
\varrho_{B}(\bar{x})=\bigcup\left\{\varrho(q)(\bar{x}, \bar{b}): q \in S\left(\operatorname{acl}^{\mathrm{eq}}(A)\right), \bar{b} \models q, \bar{b} \in B\right\} .
$$

The following notions are at the centre of [6]. We say that a theory $T a d-$ mits strongly determined types over $A(A \subset \mathbf{C})$ if every type of $S(A)$ extends to a strongly determined one. A theory $T$ admits strongly determined types if it admits strongly determined types over every set $A \subset \mathbf{C}$ of parameters.

The following lemma shows that in fact the definition of strongly determined types does not change if we strengthen it by the requirement that $\varrho(q)(\bar{x}, \bar{y})$ is a type over $\operatorname{acl}^{\mathrm{eq}}(A)$. This lemma is a version of Lemma 1.3 from $[6]$.

Lemma 1.1. Let $A \subset \mathbf{C}, A \subseteq \mathcal{A} \subseteq \operatorname{acl}^{\mathrm{eq}}(A)$ and let $\varrho(\bar{x})$ be a strongly determined type over $\mathcal{A}$. Then there exists a unique strongly determined type $\varrho^{\prime}(\bar{x})$ over $\operatorname{acl}^{\mathrm{eq}}(A)$ such that for any $q \in S\left(\operatorname{acl}^{\mathrm{eq}}(A)\right), \varrho^{\prime}(q) \vdash \varrho(q)$.

Proof. Let $M$ be very rich over $A$ : for all $n \in \omega$ and all $\bar{m} \in M, M$ realizes all $n$-types from $S\left(\operatorname{acl}^{\mathrm{eq}}(A \bar{m})\right)$. Let $\bar{c} \models \varrho_{M}$. For $q \in S\left(\operatorname{acl}^{\mathrm{eq}}(A)\right)$ and $\bar{b}=q, \bar{b} \in M$, define $\varrho^{\prime}(q):=\operatorname{tp}\left(\bar{c} \bar{b} / \operatorname{acl}^{\mathrm{eq}}(A)\right)$.

To see that the definition is correct, suppose that $\bar{b}, \bar{b}^{\prime}$ realize the same type over $\operatorname{acl}^{\text {eq }}(A)$. We may assume that $\bar{b}^{\prime} \in M$. Let $\bar{b} \bar{d} \in M$ be of the same strong type over $A$ as $\bar{b} \bar{c}$. Choose $\bar{d}^{\prime} \in M$ such that $\bar{b}^{\prime} \bar{d}^{\prime}$ is of the same strong type over $A$ as $\bar{b} \bar{d}$. Then $\varrho\left(\operatorname{tp}\left(\bar{b} \bar{d} / \operatorname{acl}^{\mathrm{eq}}(A)\right)\right)=\varrho\left(\operatorname{tp}\left(\bar{b}^{\prime} \bar{d}^{\prime} / \operatorname{acl}^{\mathrm{eq}}(A)\right)\right)$. Let $E(\bar{y}, \bar{z})$ (where $|\bar{y}|=|\bar{b}|+|\bar{c}|$ ) be a finite equivalence relation definable over $A$. Since $E(\bar{b} \bar{c}, \bar{b} \bar{d})$, we have $E\left(\overline{b^{\prime}} \bar{c}, \bar{b}^{\prime} \bar{d}^{\prime}\right)$. It follows by transitivity that $E\left(\bar{b} \bar{c}, \overline{b^{\prime}} \bar{c}\right)$. As a result $\bar{b} \bar{c}$ and $\overline{b^{\prime}} \bar{c}$ have the same strong type over $A$. The rest is easy.

It is clear from the proof that any strongly determined type $\varrho$ over $\mathcal{A}$ is determined by $\varrho_{M}$, where $M$ is very rich over $A$. 
LEMMA 1.2. Let $A \subset \mathbf{C}$ and let $T$ admit strongly determined $n$-types over a set $A$. Then every strong $n$-type over $A$ extends to a strongly determined type over $A$.

Proof. Let $q(\bar{x})$ be a type over $\operatorname{acl}^{\mathrm{eq}}(A)$ extending $p(\bar{x})$, a complete type over $A$. Let $\varrho(\bar{x})$ be a strongly determined extension of $p(\bar{x})$ and $\varrho^{\prime}(\bar{x})$ be a strongly determined type over $\operatorname{acl}^{\mathrm{eq}}(A)$ defined for $\varrho$ by Lemma 1.1. Let $q_{1}(\bar{x})$ be the strong type over acl ${ }^{\mathrm{eq}}(A)$ defined by $\varrho^{\prime}$ (by $\varrho_{M}^{\prime}$ for a saturated model $M)$. Since $q_{1}$ extends $p$, there is an automorphism $\alpha$ over $A$ taking $q_{1}$ to $q$. Let $\alpha$ take $\varrho$ to $\varrho_{1}$ (defined by $\alpha(\bar{c})$ for $\bar{c} \models \varrho_{M}$ for sufficiently saturated $M)$. Then $\varrho_{1}$ is a strongly determined extension of $q$.

The following is Lemma 2.1 from [6].

LemMA 1.3. Let $A \subseteq M$, and suppose that $M$ is very rich over $A$. Let $p(\bar{x}) \in S(A)$. Then the following are equivalent:

(i) $p$ extends to a strongly determined type over $A$;

(ii) for every finite set $\Gamma$ of finite partial $\mathrm{acl}^{\mathrm{eq}}(A)$-elementary maps $M \rightarrow M$ there exists $\bar{c} \in p(M)$ such that all maps in $\Gamma$ are elementary over $\bar{c} A$.

Our final remark here is folklore. For example it appears in some form in $[5]$.

Proposition 1.4. If a theory $T$ admits strongly determined types then $T$ is G-compact and its Lascar strong types coincide with (Shelah) strong types.

Proof. Let $M$ and $N$ be small models of $T$. We fix some enumerations of $M$ and $N$ and assume that the corresponding sequences have the same strong type. For any formula $\phi\left(x_{1}, \ldots, x_{n}\right) \in \operatorname{tp}(M)$ find a strongly determined type $\varrho$ containing $\phi$. Let $\bar{c} \models \varrho_{M N}$. Then $M$ and $N$ have the same type over $\bar{c}$. By compactness there is a model $C$ such that $M$ and $N$ have the same type over $C$. This means that $M$ and $N$ have the same Lascar strong type.

This proposition shows that admitting strongly determined types can be considered as a very strong version of G-compactness. In fact our main results below describe some situations when G-compactness implies admitting strongly determined types.

1.2. Strongly determined types and type-definable equivalence relations. Let $A \subset \mathbf{C}, A \subseteq \mathcal{A} \subseteq \operatorname{acl}^{\mathrm{eq}}(A)$ and let $\varrho$ be a strongly determined type over $\mathcal{A}$. Let $M$ be sufficiently saturated, $A \subseteq M, \bar{b} \models \varrho_{M}$ and $e$ be a finite equivalence relation definable over $\mathcal{A} \bar{b}\left({ }^{4}\right)$. The group $\operatorname{Aut}(\mathbf{C} / \bar{b} M)$ naturally acts on the set of all $e$-classes. Let $\widetilde{e}$ be the equivalence relation defined

$\left({ }^{4}\right)$ By an $\operatorname{Aut}(\mathbf{C} / \mathcal{A} \bar{b})$-invariant formula with parameters from $\mathbf{C}$. 
on the appropriate $\mathbf{C}^{l}$ by the condition that $\left(\bar{a}, \bar{a}^{\prime}\right) \in \widetilde{e}$ if and only if the corresponding $e$-classes of $\bar{a}$ and $\bar{a}^{\prime}$ are in the same orbit with respect to $\operatorname{Aut}(\mathbf{C} / \bar{b} M)$. By the choice of $\bar{b}$ any automorphism from $\operatorname{Aut}_{\mathrm{Sh}}(M / A)=$ $\operatorname{Aut}\left(M / \operatorname{acl}^{\mathrm{eq}}(A)\right)$ extends to an element of $\operatorname{Aut}\left(\mathbf{C} / \bar{b}\{M\} \operatorname{acl}^{\mathrm{eq}}(A)\right.$ ) (fixing $M$ setwise). We have obtained the exact sequence

$$
0 \rightarrow \operatorname{Aut}(\mathbf{C} / \bar{b} M) \rightarrow \operatorname{Aut}\left(\mathbf{C} / \bar{b}\{M\} \operatorname{acl}^{\mathrm{eq}}(A)\right) \rightarrow \operatorname{Aut}_{\mathrm{Sh}}(M / A) \rightarrow 0
$$

where the kernel $\operatorname{Aut}(\mathbf{C} / \bar{b} M)$ preserves all $\widetilde{e}$-classes. Hence we infer that $\operatorname{Aut}\left(\mathbf{C} / \bar{b}\{M\} \operatorname{acl}^{\mathrm{eq}}(A)\right)$ induces an action of $\operatorname{Aut}_{\mathrm{Sh}}(M / A)$ on the set of $\widetilde{e}-$ classes.

Since $e$ is a finite equivalence relation the pointwise stabilizer of the set of $\widetilde{e}$-classes is a normal subgroup $H_{e} \triangleleft \operatorname{Aut}_{\mathrm{Sh}}(M / A)$ of finite index.

Definition 1.5. We say that $\varrho$ is $G$-trivial if for any structure $M$ which realizes all strong types over $A$ and is $\operatorname{Aut}_{\mathrm{Sh}}(M / A)$-homogeneous for finite maps, and any finite equivalence relation $e$ as above, there is no $\operatorname{Aut}_{\mathrm{Sh}}(M / A)$ invariant equivalence relation $E$ properly refining $E_{\mathrm{Sh}}^{A, l}$ on the appropriate $M^{l}$ such that the corresponding group $H_{e}$ preserves all $E$-classes (in other words, for any strong type over $A$ the action of $H_{e}$ on the set of its $M$-realizations is transitive).

It is worth noting that if an equivalence relation $E$ witnesses non-Gtriviality of $\varrho$ then each $E_{\mathrm{Sh}}^{A, l}$-class on $M^{l}$ consists of $\leq\left|\operatorname{Aut}_{\mathrm{Sh}}(M / A): H_{e}\right|$ classes of $E$.

Remark. For any $\alpha \in \operatorname{Aut}(M / \mathcal{A})$ the image of a G-trivial strongly determined type is G-trivial. Thus the natural version of Lemma 1.2 for G-trivial strongly determined types still holds (by inspection of the proof).

The proposition below gives three reasons why G-trivial strongly determined types are quite frequent. For ease of notation we restrict ourselves to the case when $\mathcal{A}=A \subset \mathbf{C}$. We need the following notion.

We say that a strongly determined type $\varrho$ over $A$ is type-definable if for any $q(\bar{y}) \in S\left(\operatorname{acl}^{\mathrm{eq}}(A)\right)$ and $\phi(\bar{x}, \bar{y}) \in \varrho(q)$ there is $\psi(\bar{y}) \in q(\bar{y})$ such that for any $r(\bar{y}) \in S\left(\operatorname{acl}^{\mathrm{eq}}(A)\right)$ containing $\psi(\bar{y})$ the formula $\phi(\bar{x}, \bar{y})$ belongs to $\varrho(r)$.

Proposition 1.6. Let $A \subset \mathbf{C}$ be a countable set and $M$ be an $\operatorname{Aut}_{\text {Sh }}(M / A)$-homogeneous structure realizing all strong types over $A$.

(1) Assume that for all $l \in \omega$ every $\operatorname{Aut}_{\mathrm{Sh}}(M / A)$-invariant equivalence relation $E \subseteq M^{2 l}$ dividing each $E_{\mathrm{Sh}}^{A, l}$-class into finitely many $E$ classes is type-definable over $\operatorname{acl}^{\mathrm{eq}}(A)$. Then every strongly determined type over $A$ is $G$-trivial.

(2) If $E_{\mathrm{L}}^{A}=E_{\mathrm{KP}}^{A}$ on finite tuples (in particular if $\operatorname{Th}(M)$ is simple), then every strongly determined type over $A$ is G-trivial.

(3) A type-definable strongly determined type over $A$ is G-trivial. 
Proof. (1) Let

$$
p(\bar{x})=\left\{\phi_{0}(\bar{x}), \phi_{1}(\bar{x}), \ldots, \phi_{j}(\bar{x}), \ldots\right\}
$$

be a strong type over $A$ which is divided by $E$ into finitely many classes and let

$$
\Psi=\left\{\psi_{0}(\bar{x}, \bar{y}), \psi_{1}(\bar{x}, \bar{y}), \ldots, \psi_{j}(\bar{x}, \bar{y}), \ldots\right\}
$$

be the type over acleq $(A)$ defining $E$. We may assume that each $\phi_{j+1}(\bar{x})$ implies $\phi_{j}(\bar{x})$ and each $\psi_{j+1}(\bar{x}, \bar{y})$ implies $\psi_{j}(\bar{x}, \bar{y})$. Moreover by compactness we may assume that each $\psi_{j}(\bar{x}, \bar{y})$ is symmetric and each formula $\psi_{j+1}(\bar{x}, \bar{y}) \wedge$ $\psi_{j+1}(\bar{y}, \bar{z})$ implies $\psi_{j}(\bar{x}, \bar{z})$.

Since $p / E$ is finite, there is $j$ such that $p(\bar{x}) \cup p(\bar{y})$ implies $\psi_{j}(\bar{x}, \bar{y}) \rightarrow$ $\psi_{j+1}(\bar{x}, \bar{y})$. By compactness some $\phi_{i}(\bar{x}) \wedge \phi_{i}(\bar{y})$ implies $\psi_{j}(\bar{x}, \bar{y}) \rightarrow \psi_{j+1}(\bar{x}, \bar{y})$. We may also assume that for some $n$ the formula $\phi_{i}\left(\bar{x}_{1}\right) \wedge \cdots \wedge \phi_{i}\left(\bar{x}_{n}\right)$ implies $\bigvee\left\{\psi_{j}\left(\bar{x}_{k}, \bar{x}_{l}\right): 0 \leq k<l \leq n\right\}$. Thus $\psi_{j}$ defines a finite equivalence on $\phi_{i}$ which coincides with $E$ on $p(\mathbf{C})$. This contradicts the assumption that $p$ is a strong type.

As a result we see that there is no $\operatorname{Aut}_{\mathrm{Sh}}(M / A)$-invariant equivalence relation $E$ properly dividing some $E_{\mathrm{Sh}}^{A}$-class into finitely many $E$-classes. This means that every strongly determined type over $A$ satisfies the definition of G-trivial types.

(2) Let $M$ be sufficiently saturated. That $\operatorname{Aut}_{\mathrm{Sh}}(M / A) / \operatorname{Aut}_{\mathrm{KP}}(M / A)$ is a compact connected group is folklore (for example it can be deduced from Lemma 4.10 of [10] or Remark 3.1 from [8]). A complete proof of this is given in Theorem 21 of [14]. By a theorem of Mycielski from [11] the group $\operatorname{Aut}_{\mathrm{Sh}}(M / A) / \operatorname{Aut}_{\mathrm{KP}}(M / A)$ is divisible and does not have subgroups of finite index. On the other hand, if $E$ is an equivalence relation as in the definition of G-trivial strongly determined types, then it is coarser than the corresponding $E_{\mathrm{KP}}^{A, l}\left(=E_{\mathrm{L}}^{A, l}\right)$. This implies that the subgroup $H<\operatorname{Aut}_{\mathrm{Sh}}(M / A)$ fixing all $E$-classes is a proper subgroup of $\operatorname{Aut}_{\mathrm{Sh}}(M / A)$ of finite index which contains $\operatorname{Aut}_{\mathrm{KP}}(M / A)$. This contradiction shows that every strongly determined type over $A$ is $\mathrm{G}$-trivial.

(3) Let $\varrho$ be a strongly determined type over $A, \bar{b}=\varrho_{M}$ and $e$ be a finite equivalence relation definable over $A \bar{b}$. As above define the corresponding equivalence relation $\widetilde{e} \supset e$. The pointwise stabilizer of the set of $\widetilde{e}$-classes forms a normal subgroup $H_{e} \triangleleft \operatorname{Aut}_{\mathrm{Sh}}(M / A)$ of finite index.

For any $\widetilde{e}$-class find a formula $\theta(\bar{u}, \bar{b}, \bar{d})$ with $\bar{d} \in M$ asserting that the tuple $\bar{u}$ represents one of the $e$-classes of this class. If $\bar{d}^{\prime} \in M$ is of the same strong type over $A$ as $\bar{d}$ then by the choice of $\bar{b}$ and (the proof of) Lemma 1.1, $\operatorname{tp}\left(\overline{d b} / \operatorname{acl}^{\mathrm{eq}}(A)\right)=\operatorname{tp}\left(\overline{d^{\prime}} \bar{b} / \mathrm{acl}^{\mathrm{eq}}(A)\right)$ and we see that $\theta\left(\bar{u}, \bar{b}, \bar{d}^{\prime}\right)$ still describes $e$-classes of some $\widetilde{e}$-class. Below we assume that all $\widetilde{e}$-classes are so defined over $\bar{b} \bar{d}$ by appropriate formulas. 
We see that the equivalence relation of $H_{e^{-}}$orbits on the $\operatorname{Aut}_{S h}(M / A)$ orbit of $\bar{d}$ is defined by the formula over $A \bar{b}$ which is the conjunction $\Phi\left(\bar{w}^{\prime}, \bar{w}^{\prime \prime}, \bar{b}\right)$ of the conditions of the form $\forall \bar{u}\left(\theta\left(\bar{u}, \bar{b}, \bar{w}^{\prime}\right) \leftrightarrow \theta\left(\bar{u}, \bar{b}, \bar{w}^{\prime \prime}\right)\right.$ ) (for all $\widetilde{e}$-classes $)$. Since $\bar{b} \models \varrho_{M}$, the truth of $\Phi\left(\bar{d}^{\prime}, \bar{d}^{\prime \prime}, \bar{b}\right)$ is determined by the strong type of $\bar{d}^{\prime} \bar{d}^{\prime \prime}$ over $A$. This means that $\neg \Phi\left(\bar{w}^{\prime}, \bar{w}^{\prime \prime}, \bar{b}\right)$ for tuples from $M$ of the type $\operatorname{tp}\left(\bar{d} / \mathrm{acl}^{\mathrm{eq}}(A)\right)$ is equivalent to some disjunction of strong types on $\bar{w}^{\prime} \bar{w}^{\prime \prime}$ over $A$.

On the other hand, since $\varrho$ is type-definable, any strong type of this form contains a formula over acl ${ }^{\mathrm{eq}}(A)$ which implies $\neg \Phi\left(\bar{w}^{\prime}, \bar{w}^{\prime \prime}, \bar{b}\right)$. We now see that $\Phi\left(\bar{w}^{\prime}, \bar{w}^{\prime \prime}, \bar{b}\right)$ is equivalent to a conjunction of formulas over $\operatorname{acl}^{\mathrm{eq}}(A)$. As a result we have obtained an equivalence relation type-definable over $\operatorname{acl}^{\text {eq }}(A)$ which divides $\operatorname{stp}(\bar{d} / A)$ into finitely many classes. The argument of the first part of the proposition shows that the number of classes is 1 . Thus $\theta(\mathbf{C}, \bar{b}, \bar{d})=\theta\left(\mathbf{C}, \bar{b}, \bar{d}^{\prime}\right)$ for $\theta$ as above and $\bar{d}^{\prime}$ of the same strong type over $A$ as $\bar{d}$. This shows that $\varrho$ is G-trivial.

REMARK. It is worth noting that in the definition of G-triviality it only suffices to demand that the corresponding property holds over some fixed model $M$ (saturated and homogeneous enough). Indeed, let $\varrho$ be a strongly determined type over $A, M<M^{\prime}$ be sufficiently saturated and homogeneous models, $\bar{b}=\varrho_{M}, \bar{b}^{\prime} \models \varrho_{M^{\prime}}$ and $\phi(\bar{x}, \bar{y}, \bar{z})$ be a formula over $A$ such that $\phi(\bar{x}, \bar{y}, \bar{b})$ defines a finite equivalence relation $e$. As above define the corresponding equivalence relation $\widetilde{e} \supset e$. The pointwise stabilizer of the set of $\widetilde{e}$-classes forms a normal subgroup $H_{e} \triangleleft \operatorname{Aut}_{\mathrm{Sh}}(M / A)$ of finite index.

Assume that the definition of G-triviality holds for $M$ and $\bar{b}$. For any $\widetilde{e}-$ class find a formula $\theta(\bar{u}, \bar{b}, \bar{d})$ with $\bar{d} \in M$ asserting that the tuple $\bar{u}$ represents one of the $e$-classes of this $\widetilde{e}$-class. If $\bar{d}^{\prime} \in M$ is of the same strong type over $A$ as $\bar{d}$ then by the choice of $\bar{b}, \operatorname{tp}\left(\overline{d b} / \mathrm{acl}^{\mathrm{eq}}(A)\right)=\operatorname{tp}\left(\bar{d}^{\prime} \bar{b} / \mathrm{acl}^{\mathrm{eq}}(A)\right)$ and by G-triviality of $\varrho$ over $M$ we deduce that $\theta\left(\bar{u}, \bar{b}, \bar{d}^{\prime}\right)$ still describes $e$-classes of the same $\widetilde{e}$-class.

If now $\bar{d}^{\prime}$ belongs to $M^{\prime}$ and is of the same strong type over $A$ as $\bar{d}$, then

$$
\operatorname{tp}\left(\overline{d b} / \mathrm{acl}^{\mathrm{eq}}(A)\right)=\operatorname{tp}\left(\overline{d b}^{\prime} / \mathrm{acl}^{\mathrm{eq}}(A)\right)=\operatorname{tp}\left(\bar{d}^{\prime} \bar{b}^{\prime} / \mathrm{acl}^{\mathrm{eq}}(A)\right) .
$$

This implies that for $e^{\prime}$ defined by $\phi\left(\bar{x}, \bar{y}, \bar{b}^{\prime}\right)$ the formulas $\theta\left(\bar{u}, \bar{b}^{\prime}, \bar{d}^{\prime}\right)$ and $\theta\left(\bar{u}, \bar{b}^{\prime}, \bar{d}\right)$ describe $e^{\prime}$-classes of the same $\widetilde{e}^{\prime}$-class (the strong type of $\bar{b}^{\prime} \bar{d} \bar{d}^{\prime}$ over $A$ coincides with the type of some $\bar{b} \bar{d} \bar{d}^{\prime \prime}$ with $\bar{d}^{\prime \prime} \in M$ of the type $\left.\operatorname{stp}(\bar{d} / A)\right)$. This implies G-triviality with respect to $M^{\prime}$. The rest is obvious.

Question. Is it possible that in the situation when $M$ is sufficiently saturated and homogeneous, the group $\operatorname{Aut}_{\mathrm{KP}}(M)$ has a subgroup of finite index $G$ such that the $G$-orbit equivalence relation on some $M^{k}$ properly refines $E_{\mathrm{KP}}^{k}$ ? 
2. G-compactness and strongly determined types. Our main result in this section gives some sufficient conditions (in terms of strongly determined types) for $E_{\mathrm{L}}=E_{\mathrm{Sh}}$. Then we study to what extent G-compactness is needed for existence of strongly determined $n$-types when $n>1$. The following statement corrects Lemma 2.2 from [6] by adding the assumption that $E_{\mathrm{L}}=E_{\mathrm{KP}}$ on finite tuples. The idea that Lemma 2.2 should have some assumption connected with G-compactness was suggested by E. Hrushovski.

THEOREM 2.1. Let $T$ be a complete theory such that for any finite $A$ of the basic sort, $E_{\mathrm{L}}^{A}=E_{\mathrm{KP}}^{A}$ on finite tuples, and every 1-type over $A$ extends to a strongly determined type over $A$. Then for all tuples $\bar{a}$ and all $n>0$, every $n$-type of $T$ over $\bar{a}$ has a strongly determined extension over $\bar{a}$. Moreover, all strongly determined types over $\bar{a}$ are G-trivial.

This theorem implies that if $T$ is simple and every 1-type over a finite set extends to a strongly determined type, then any $n$-type over a finite set extends to a strongly determined one. The proof is based on the following theorem.

THEOREM 2.2. Let $T$ be a complete theory such that for any finite set $A$ of the basic sort, $E_{\mathrm{L}}^{A}=E_{\mathrm{KP}}^{A}$ on finite tuples, and every 1-type over $A$ extends to a strongly determined type over $A$. Then for all finite sets $A$ of the basic sort, $E_{\mathrm{L}}^{A}=E_{\mathrm{Sh}}^{A}$ on finite tuples. In particular, $E_{\mathrm{KP}}^{A, k}=E_{\mathrm{Sh}}^{A, k}$ for all $A$ and $k \leq \omega$.

Proof. The last statement of the theorem follows from the main statement by the definition of $E_{\mathrm{KP}}^{A}$ and $E_{\mathrm{Sh}}^{A}$ (for example as in the proof of Theorem 15 from [7]).

Let $\Psi(\bar{x}, \bar{y})$ be a type defining $E_{\mathrm{L}}^{\bar{a}}$ on $n$-tuples satisfying $p(\bar{x}) \in S(\bar{a})$. We may assume that

$$
\begin{aligned}
p(\bar{x}) & =\left\{\phi_{0}(\bar{x}), \phi_{1}(\bar{x}), \ldots, \phi_{j}(\bar{x}), \ldots\right\}, \\
\Psi & =\left\{\psi_{0}(\bar{x}, \bar{y}), \psi_{1}(\bar{x}, \bar{y}), \ldots, \psi_{j}(\bar{x}, \bar{y}), \ldots\right\},
\end{aligned}
$$

where each $\phi_{j+1}(\bar{x})$ implies $\phi_{j}(\bar{x})$ and each $\psi_{j+1}(\bar{x}, \bar{y})$ implies $\psi_{j}(\bar{x}, \bar{y})$. We want to prove that $\Psi(\bar{x}, \bar{y})$ does not refine $E_{\mathrm{Sh}}^{\bar{a}, n}$ on $p(\mathbf{C})$. By Proposition 1.6(2) we assume below that every 1-type over $\bar{a}$ extends to a G-trivial strongly determined type over $\bar{a}$.

The proof is by induction on $|\bar{x}|$. Let $|\bar{x}|=1$. By Lemma 1.2 any strong type over $\bar{a}$ extending $p(x)$ extends to a strongly determined type. Let $\varrho(x)$ be such a type. Let $c \models p(x)$ be of the strong type defined by $\varrho$. If $\varrho_{c}(x)$ contains $\neg \psi_{j}(x, c)$ for some $j$, then for any set $C \subset p(\mathbf{C})$ consisting of elements of the same strong type as $c$, any realization of $\varrho_{M}$ for a sufficiently saturated structure $M \supset C$ provides an element not $E_{\mathrm{L}}$-equivalent to any element of $C$. This contradicts the boundedness of $E_{\mathrm{L}}$. 
We see that $\varrho_{c}(x)$ contains all $\psi_{j}(x, c) \wedge \phi_{j}(x)$. Then by the definition of a strongly determined type for any $c^{\prime}$ of the same strong type as $c$ the type $\varrho_{c^{\prime}}(x)$ contains all formulas $\psi_{j}\left(x, c^{\prime}\right) \wedge \phi_{j}(x)$. Let $b \models \varrho_{M}(x)$, where $c, c^{\prime} \in M$. Then $(c, b)$ and $\left(c^{\prime}, b\right)$ are in $E_{\mathrm{L}}$. Thus $\left(c, c^{\prime}\right) \in E_{\mathrm{L}}$.

Towards a contradiction assume that for some finite $A, E_{\mathrm{L}}^{A} \neq E_{\mathrm{Sh}}^{A}$ on finite tuples. Choose $p(\bar{x})$ and $\bar{a}$ as above so that $n=|\bar{x}|$ is minimal with $E_{\mathrm{L}}^{\bar{a}, n} \neq E_{\mathrm{Sh}}^{\bar{a}, n}$ on tuples satisfying $p(\bar{x})$. Then $n>1$. We may assume that $p(\bar{x})$ is not algebraic with respect to the first coordinate. Since we assume $E_{\mathrm{L}}^{\bar{a}, n} \neq E_{\mathrm{Sh}}^{\bar{a}, n}$ on $p(\mathbf{C})$, each $E_{\mathrm{Sh}^{-}}$class from $p(\mathbf{C})$ splits into several $E_{\mathrm{L}^{-}}$ classes.

Let $p_{0}\left(x_{1}\right)$ be a non-algebraic type over $\bar{a}$ containing all formulas of the form $\exists x_{2}, \ldots, x_{n} \phi_{j}(\bar{x})$. Then by an appropriate version of Lemma 1.2 any strong type over $\bar{a}$ extending $p_{0}\left(x_{1}\right)$ extends to a G-trivial strongly determined type. Fix such a strong type and take an appropriate $\varrho\left(x_{1}\right)$. Let $M$ contain representatives of all $E_{\mathrm{L}}^{\bar{a}, n}$-classes and $b_{1} \models \varrho_{M}$. Take a strong type $q_{1}\left(x_{1}, \ldots, x_{n}\right) \in S\left(\operatorname{acl}^{\mathrm{eq}}(\bar{a})\right)$ extending $p(\bar{x}) \cup \operatorname{stp}\left(b_{1} / \bar{a}\right)$ and find $b_{2}, \ldots, b_{n}$ such that $q_{1}(\bar{x})=\operatorname{stp}\left(b_{1} b_{2} \ldots b_{n} / \bar{a}\right)$. Since any strong type over $\bar{a}$ extending $p(\bar{x})$ can be chosen as $q_{1}$ for appropriate $\varrho\left(x_{1}\right)$, the proof of the theorem will be finished if we show that $q_{1}(\mathbf{C})$ does not split into several $E_{\mathrm{L}}$-classes.

Let $q_{1}(\bar{x})=\left\{\phi_{0}^{\prime}, \ldots, \phi_{i}^{\prime}, \ldots\right\}$ with $\phi_{i+1}^{\prime} \vdash \phi_{i}^{\prime}, i \in \omega$. Here we assume that each $\phi_{i}^{\prime}$ defines an equivalence class of some finite equivalence relation over $\bar{a}$. Below we present all $\phi_{i}^{\prime}$ by formulas over parameters from $M$ with $\exists x_{2}, \ldots, x_{n} \phi_{i}^{\prime}(\bar{x}) \in \operatorname{tp}\left(b_{1} / M\right)$ under that presentation. We will use the fact that any automorphism of $M$ strong over $\bar{a}$ fixes $\phi_{i}^{\prime}(M)$.

Let $q_{2}\left(x_{2}, \ldots, x_{n}\right)=\operatorname{stp}\left(b_{2} \ldots b_{n} / b_{1} \bar{a}\right)=\left\{\phi_{0}^{\prime \prime}, \ldots, \phi_{i}^{\prime \prime}, \ldots\right\}$ with $\phi_{i+1}^{\prime \prime} \vdash \phi_{i}^{\prime \prime}$, $i \in \omega$. We assume that each $\phi_{i}^{\prime \prime}$ defines an equivalence class of a finite equivalence relation defined by a formula $e_{i}\left(\bar{u}, \bar{v}, b_{1}, \bar{a}\right)$ over $b_{1} \bar{a}$. We also assume that $\phi_{i}^{\prime}(\bar{x})$ implies that the equivalence relation $e_{i}\left(\bar{u}, \bar{v}, x_{1}, \bar{a}\right)$ over $x_{1} \bar{a}$ is finite.

The group Aut $\left(\mathbf{C} / b_{1} M\right)$ naturally acts on the set of all $e_{i}$-classes. Let $\widetilde{e}_{i} \supset e_{i}$ be the equivalence relation defined by: $\left(\bar{c}_{1}, \bar{c}_{2}\right) \in \widetilde{e}$ if the $e_{i}$-classes of $\bar{c}_{1}$ and $\bar{c}_{2}$ are in the same $\operatorname{Aut}\left(\mathbf{C} / b_{1} M\right)$-orbit. For the $\operatorname{Aut}\left(\mathbf{C} / b_{1} M\right)$-orbit of the $e_{i}$-class corresponding to $\phi_{i}^{\prime \prime}$, find a formula $\theta_{i}\left(\bar{u}, b_{1}, \bar{d}_{i}\right) \in \operatorname{tp}\left(b_{2} \ldots b_{n} / b_{1} M\right)$ with $\bar{d}_{i} \in M$ asserting that $\bar{u}$ represents one of the $e_{i}$-classes of this orbit. If $\bar{d}_{\underline{i}}^{\prime} \in M$ is of the same strong type over $\bar{a}$ as $\bar{d}_{i}$ then by the choice of $b_{1}$, $\operatorname{tp}\left(\bar{d}_{i} b_{1} / \operatorname{acl}^{\mathrm{eq}}(\bar{a})\right)=\operatorname{tp}\left(\bar{d}_{i}^{\prime} b_{1} / \mathrm{acl}^{\mathrm{eq}}(\bar{a})\right)$ and $\theta_{i}\left(\bar{u}, b_{1}, \bar{d}_{i}^{\prime}\right)$ still describes an orbit of $e_{i}$-classes (i.e. defines an $\widetilde{e}_{i}$-class). Extending $\bar{d}_{i}$ if necessary, we may assume that all $\widetilde{e}_{i}$-classes are defined over $b_{1} \bar{d}_{i}$ by appropriate formulas. Since $\varrho$ is G-trivial we find that

$$
\begin{aligned}
& \text { for any } \bar{d}_{i}^{\prime} \in M \text { of the same strong type over } \bar{a} \text { as } \bar{d}_{i}, \\
& \theta_{i}\left(\mathbf{C}, b_{1}, \bar{d}_{i}\right)=\theta_{i}\left(\mathbf{C}, b_{1}, \bar{d}_{i}^{\prime}\right) \text {. }
\end{aligned}
$$


Let $\bar{c}=\left(c_{1}, c_{2}, \ldots, c_{n}\right) \in M$ be of the same strong type as $\bar{b}$ over $\bar{a}$. If for some $j$ the type $\operatorname{tp}\left(b_{1} / M\right)$ contains

$$
\forall x_{2}, \ldots, x_{n}\left(\phi_{j}^{\prime}(\bar{x}) \wedge \theta_{j}\left(x_{2}, \ldots, x_{n}, x_{1}, \bar{d}_{j}\right) \rightarrow \neg \psi_{j}(\bar{x}, \bar{c})\right)
$$

then for any $\bar{c}^{\prime} \subset M$ with $\operatorname{stp}(\bar{c} / \bar{a})=\operatorname{stp}\left(\bar{c}^{\prime} / \bar{a}\right)$ and for appropriate $\bar{d}_{j}^{\prime} \in M$ of the same strong type as $\bar{d}_{j}$ over $\bar{a}$ we have

$$
\forall x_{2}, \ldots, x_{n}\left(\phi_{j}^{\prime}(\bar{x}) \wedge \theta_{j}\left(x_{2}, \ldots, x_{n}, x_{1}, \bar{d}_{j}^{\prime}\right) \rightarrow \neg \psi_{j}\left(\bar{x}, \bar{c}^{\prime}\right)\right) \in \operatorname{tp}\left(b_{1} / M\right) .
$$

By the previous paragraph,

$$
\forall x_{2}, \ldots, x_{n}\left(\phi_{j}^{\prime}(\bar{x}) \wedge \theta_{j}\left(x_{2}, \ldots, x_{n}, x_{1}, \bar{d}_{j}\right) \rightarrow \neg \psi_{j}\left(\bar{x}, \bar{c}^{\prime}\right)\right) \in \operatorname{tp}\left(b_{1} / M\right) .
$$

Thus $b_{1} \ldots b_{n}$ is not $E_{\mathrm{L}^{-}}$equivalent to any $\bar{c}^{\prime} \subset M$ with $\operatorname{stp}(\bar{b} / \bar{a})=\operatorname{stp}\left(\bar{c}^{\prime} / \bar{a}\right)$. This contradicts the choice of $M$.

We see that for every $j$ the type $\operatorname{tp}\left(b_{1} / M\right)$ contains all

$$
\exists x_{2}, \ldots, x_{n}\left(\phi_{j}^{\prime}(\bar{x}) \wedge \theta_{j}\left(x_{2} \ldots x_{n}, x_{1}, \bar{d}_{j}\right) \wedge \psi_{j}\left(\bar{x}, \bar{c}^{\prime}\right)\right)
$$

with $\operatorname{stp}(\bar{c} / \bar{a})=\operatorname{stp}\left(\bar{c}^{\prime} / \bar{a}\right), \bar{c}^{\prime} \subset M$.

We now claim that for every $\bar{c}^{\prime}$ as above, there is a realization of all

$$
\phi_{j}^{\prime}\left(b_{1}, x_{2} \ldots x_{n}\right) \wedge e_{j}\left(x_{2} \ldots x_{n}, b_{2} b_{3} \ldots b_{n}, b_{1} \bar{a}\right) \wedge \psi_{j}\left(b_{1} x_{2} \ldots x_{n}, \bar{c}^{\prime}\right) .
$$

To see this we apply compactness and the following argument. If $b_{2}^{\prime} \ldots b_{n}^{\prime}$ realizes $\phi_{j}^{\prime}\left(b_{1}, x_{2} \ldots x_{n}\right) \wedge \theta_{j}\left(x_{2} \ldots x_{n}, b_{1}, \bar{d}_{j}\right) \wedge \psi_{j}\left(b_{1} x_{2} \ldots x_{n}, \bar{c}^{\prime}\right)$, then by the definition of $\theta$, there is $\gamma \in \operatorname{Aut}\left(\mathbf{C} / b_{1} M\right)$ taking the $e_{j}$-class of $b_{2}^{\prime} \ldots b_{n}^{\prime}$ to the $e_{j}$-class of $b_{2} \ldots b_{n}$. Then $\gamma\left(b_{2}^{\prime}\right) \ldots \gamma\left(b_{n}^{\prime}\right)$ realizes

$$
\phi_{j}^{\prime}\left(b_{1}, x_{2} \ldots x_{n}\right) \wedge e_{j}\left(x_{2} \ldots x_{n}, b_{2} b_{3} \ldots b_{n}, b_{1} \bar{a}\right) \wedge \psi_{j}\left(b_{1} x_{2} \ldots x_{n}, \bar{c}^{\prime}\right) .
$$

Now assume that $b_{1} b_{2}^{\prime} \ldots b_{n}^{\prime}$ realizes all formulas

$$
\phi_{j}^{\prime}(\bar{x}) \wedge e_{j}\left(x_{2} \ldots x_{n}, b_{2} \ldots b_{n}, x_{1}, \bar{a}\right) \wedge \psi_{j}\left(\bar{x}, \bar{c}^{\prime}\right) .
$$

Then $b_{2} \ldots b_{n}$ and $b_{2}^{\prime} \ldots b_{n}^{\prime}$ have the same strong type over $\bar{a} b_{1}$. By induction they realize the same Lascar strong type over $b_{1} \bar{a}$. This obviously implies that $b_{1} b_{2}^{\prime} \ldots b_{n}^{\prime}$ and $b_{1} b_{2} \ldots b_{n}$ have the same Lascar strong type over $\bar{a}$. Then we see that $\bar{c}^{\prime}$ and $\bar{b}$ have the same Lascar strong type as $b_{1} b_{2}^{\prime} \ldots b_{n}^{\prime}$. Since $\bar{c}^{\prime}$ is an arbitrary realization of the strong type $q_{1}(\bar{x})$, we have a contradiction with the assumption that the corresponding $E_{\mathrm{Sh}}^{\bar{a}}$-class splits into several $E_{\mathrm{L}}^{\bar{a}}$-classes.

Proposition 2.3. Let $T$ be a complete theory such that for any finite $A$, $E_{\mathrm{L}}^{A}=E_{\mathrm{Sh}}^{A}$ on finite tuples (for example $T$ is $\omega$-categorical), and every 1-type over $A$ extends to a strongly determined type over $A$. Then for all tuples $\bar{a}$ and all $n>0$, every $n$-type of $T$ over $\bar{a}$ has a strongly determined extension over $\bar{a}$. Moreover each strongly determined type over $\bar{a}$ is G-trivial.

Proof. Pick $M$ and $\bar{a} \in M$, and let $p(\bar{x})$ be an $n$-type of $\operatorname{Th}(M)$ over $\bar{a}$. Form an increasing chain of sufficiently saturated structures, $M_{0}:=M \prec$ 
$M_{1} \prec \cdots \prec M_{n}$, together with $c_{1}, \ldots, c_{n}$ such that for each $i<n$, if $p \not$ $x_{i} \in \operatorname{acl}\left(\bar{a}\left\{x_{j}: j<i\right\}\right)$ then $c_{i} \in M_{i} \backslash M_{i-1}$ and $c_{i}$ realizes a 1-type over $M_{i-1}$ defining a strongly determined type over $\left(c_{1}, \ldots, c_{i-1}, \bar{a}\right)$, and such that $\left(c_{1}, \ldots, c_{n}\right)$ realizes $p$.

To see that there is a strongly determined type $\varrho$ over $\bar{a}$ such that $\left(c_{1}, \ldots, c_{n}\right)$ realizes $\varrho_{M}$ it suffices to show that for any $\bar{b}, \bar{b}^{\prime} \in M$ of the same strong type over $\bar{a}$ the tuples $\bar{b}$ and $\bar{b}^{\prime}$ have the same strong type over $\bar{a} \bar{c}$ (then define $\varrho(\operatorname{stp}(\bar{b} / \bar{a})):=\operatorname{tp}(\bar{c} \bar{b} / \bar{a}))$. Having chosen $\bar{b}, \bar{b}^{\prime} \in M$ of the same strong type over $\bar{a}$ we prove by induction that for each $i$, the tuples $\bar{b}, \bar{b}^{\prime}$ have the same strong type over $\bar{a} c_{1}, \ldots, c_{i}$. The case $i=0$ is obvious.

At step $i \rightarrow i+1$ if $c_{i+1} \in \operatorname{acl}\left(\bar{a} c_{1}, \ldots, c_{i}\right)$, then $\bar{b}$ and $\bar{b}^{\prime}$ realize the same strong type over $\bar{a} c_{1}, \ldots, c_{i+1}$. Consider the case when $c_{i+1} \notin \operatorname{acl}\left(\bar{a} c_{1}, \ldots, c_{i}\right)$. Then there is a strongly determined type $\varrho^{\prime}$ over $\bar{a} c_{1}, \ldots, c_{i}$ such that $c_{i+1} \models \varrho_{M_{i}}^{\prime}$.

Since $E_{\mathrm{L}}=E_{\mathrm{Sh}}$ there are $\bar{b}_{0}(=\bar{b}), \bar{b}_{1}, \ldots, \bar{b}_{m}\left(=\bar{b}^{\prime}\right)$ such that every pair $\bar{b}_{l}, \bar{b}_{l+1}$ belongs to some infinite $\bar{a} c_{1} \ldots c_{i}$-indiscernible sequence $I_{l}, l=$ $0, \ldots, m-1$. It is clear that all ordered (according to the enumeration) pairs from $I_{l}$ have the same strong type over $\bar{a} c_{1}, \ldots, c_{i}$. Thus for any realization $d_{l} \models \varrho_{I_{l}}^{\prime}$ the tuples $\bar{b}_{l}$ and $\bar{b}_{l+1}$ have the same strong type over $\bar{a} c_{1}, \ldots, c_{i}, d_{l}$. This implies that if $d \models \varrho_{\bar{b}_{0}, \ldots, \bar{b}_{m}}^{\prime}$, then all $\bar{b}_{l}$ have the same strong type over $\bar{a} c_{1}, \ldots, c_{i}, d$. By the choice of $c_{i+1}$ we now see that the tuples $\bar{b}$ and $\bar{b}^{\prime}$ realize the same strong type over $\bar{a} c_{1}, \ldots, c_{i+1}$.

The last statement of the proposition follows from Proposition 1.6.

\section{Proof of Theorem 2.1. By Theorem 2.2 we may use Proposition 2.3.}

Lemma 2.2 in [6] was applied in a few places there to show that some theories admit strongly determined types. It has already been mentioned in [4] that most applications of Lemma 2.2 in [6] are unaffected. We can now show this by applying Theorem 2.1. For example, Theorem 2.6 of [6] states that every weakly o-minimal theory admits strongly determined types. We recall that a theory is weakly o-minimal if every definable subset of every model is a finite union of convex sets. To repair the proof given in [6] we need the following fact:

Every automorphism of a big saturated weakly o-minimal structure is Lascar strong. In particular $E_{\mathrm{L}}=E_{\mathrm{KP}}$ over finite sets.

The proof of Lemma 24 of [14] (which states the same for o-minimal theories) works without any changes. For completeness we mention that the proof is based on the following statement: for any two small submodels $M$ and $N$ of the same type over $\emptyset$ every consistent formula $\phi(\bar{z})$ has a realization $\bar{c}$ such that $M$ and $N$ have the same type over $\bar{c}$. This can be proved by induction on $|\bar{z}|$. 
We can now repeat the proof of Theorem 2.6 of [6] by replacing everywhere Lemma 2.2 by Theorem 2.1 just proved.

Another application of Theorem 2.1 is the following statement.

COROLlarY 2.4. Every $C$-minimal structure having $E_{\mathrm{KP}}=E_{\mathrm{L}}$ over finite sets admits strongly determined types.

This can be shown by following the proof of Theorem 2.10 and Remark 2.11 of [6]. In these arguments we should replace Lemma 2.2 by Theorem 2.1.

The statement of the corollary is not as strong as the corresponding one in [6]. The difference is that we now assume $E_{\mathrm{KP}}=E_{\mathrm{L}}$ over finite sets. On the other hand, many C-minimal structures satisfy this. In particular it is true for non-trivially valued algebraically closed fields where the C-relation is naturally defined from the valuation. In fact in [5] it is explicitly shown that then $E_{\mathrm{L}}^{k}=E_{\mathrm{Sh}}^{k}$ for all finite $k$ as well as that every type extends to a strongly determined one (in [4] and [5] they are called invariant types).

3. Examples of simple theories. Theorem 2.2 has some applications connected with the problem whether $E_{\mathrm{L}}=E_{\mathrm{Sh}}$ holds for simple theories. It is well known that simple teories are G-compact (for example, see [7]). Now the statement below is a consequence of Theorem 2.2 and Proposition 1.6.

Let $T$ be a simple theory such that every 1-type of $T$ over any finite set $A$ extends to a strongly determined type over $A$. Then $E_{\mathrm{L}}^{\bar{a}, n}=E_{\mathrm{Sh}}^{\bar{a}, n}$ for all $n$ and tuples $\bar{a}$.

It is unclear when a simple theory admits strongly determined (KPdetermined) types. In [6] there are examples of simple theories where a strongly determined type does not exist. On the other hand, it makes sense to verify this property for simple theories which are obtained from stable ones by adding a relation in some "generic" way.

First, we discuss one of the constructions presented in [3]. We start with a complete theory $T$ which admits elimination of quantifiers and elimination of the quantifier $\exists^{\infty}$ (for example, a complete theory of algebraically closed fields). Fix a sort $S$ of the theory and extend the language by a unary predicate $P$ of this sort. Then Theorem 2.4 from [3] states that the theory of all $P$-expansions has a model companion $T_{P, S}$. Corollary 2.8 there states that $T_{S, P}$ is simple if $T$ is simple. The following proposition concerns a number of examples of simple theories (including random graphs and generic directed graphs; see 2.12 in [3]).

Proposition 3.1. If $T$ is simple and admits strongly determined types then so does $T_{S, P}$.

Proof. Let $M \models T_{S, P}$ be sufficiently saturated and $D \subset M$. By Lemma 1.3 (Lemma 2.1 from [6]) given a type $p$ over $D$ and a finite family $\Gamma$ of finite 
$\operatorname{acl}^{\mathrm{eq}}(D)$-elementary maps in $M$ it suffices to find a realization $\bar{a}=p$ such that all maps from $\Gamma$ are elementary over $\bar{a} D$. Let $C=\bigcup\{\operatorname{Dom}(\gamma) \cup \operatorname{Rng}(\gamma)$ : $\gamma \in \Gamma$. We may assume that $D=\emptyset$. Since $T$ admits strongly determined types, find $\bar{d}$ realizing a strongly determined extension $\varrho_{M}$ of $p$ over $M$ (with respect to $T)$. Then $\bar{d}$ is forking-independent of $C$. In particular, for any $\bar{c} \in C$ (we admit the case $\bar{c}=\emptyset$ ), $\operatorname{acl}_{T}(\bar{c} \bar{d}) \cap \operatorname{acl}_{T}(C)=\operatorname{acl}_{T}(\bar{c})$. Using this we can extend the P-structure of $\operatorname{acl}_{T}(C)$ to a P-expansion on $\operatorname{acl}_{T}(\bar{d} C)$ so that any map $\bar{b} \rightarrow \bar{b}^{\prime}$ from $\Gamma$ induces a P-preserving map $\operatorname{acl}_{T}(\overline{d b}) \rightarrow$ $\operatorname{acl}_{T}\left(\overline{d b^{\prime}}\right)$ and the corresponding $P$-expansion of $\operatorname{acl}_{T}(\bar{d})$ agrees with $p$. Note that to satisfy the first condition it is enough to assume that all elements of $\operatorname{acl}_{T}(\bar{d} C) \backslash\left(\operatorname{acl}_{T}(C) \cup \operatorname{acl}_{T}(\bar{d})\right)$ are not in $P$. Since any map from $\Gamma$ acts trivially on $\operatorname{acl}_{T}(\emptyset)$, we can now take any $P$-expansion of $\operatorname{acl}_{T}(\bar{d})$ extending the $P$-structure of $\operatorname{acl}_{T}(\emptyset)$ and agreeing with $p$.

By the axioms of $T_{S, P}$ (Theorem 2.4 from [3]) given a realization $\bar{d}^{\prime}$ of an extension of the $T$-part of $p$ over $C$, the isomorphism type of any $P$-expansion of $\operatorname{acl}_{T}\left(\bar{d}^{\prime} C\right)$ extending the $P$-expansion of $\operatorname{acl}(C)$ in $M$ is realized in $M$ on some $\operatorname{acl}_{T}\left(\bar{d}^{\prime \prime} C\right)$, where $\bar{d}^{\prime \prime}$ is of the same type as $\bar{d}^{\prime}$ over $C$ with respect to $T$. Using this find $\bar{a}$ realizing on $\operatorname{acl}_{T}(\bar{a} C)$ the $P$-expansion of $\operatorname{acl}_{T}(\bar{d} C)$ built in the previous paragraph. By Corollary 2.6 from [3] the type $p$ is determined by the isomorphism type of the expanded structure on $\operatorname{acl}_{T}(\bar{a})$ (with distinguished $\bar{a} \models p$ ). Applying this we see that $\bar{a} \models p$ and any map from $\Gamma$ is elementary over $\bar{a}$.

Proposition 3.1 together with the discussion from the first paragraph of this section gives another proof of the following statement from [3] (end of Section 1).

Corollary 3.2. Let $T$ be a simple theory of the form $T_{S, P}$ as in Proposition 3.1. Then $E_{\mathrm{L}}=E_{\mathrm{Sh}}$.

The second construction from [3] assigns a model companion $T_{A}$ (if it exists) to the theory of all structures $(M, \sigma)(\sigma \in \operatorname{Aut}(M))$ for models $M$ of a complete theory $T$ which admits elimination of quantifiers and has the PAPA. Corollary 3.8 from [3] states that $T_{A}$ is simple if $T$ is stable. The theory ACFA of algebraically closed fields with a generic automorphism [2] is an example of such $T_{A}$. It looks likely that the approach of Theorem 3.1 can be developed to obtain admitting strongly determined types in the case of $T_{A}$ (or ACFA). This case is open. It is more complicated than that of Proposition 3.1 .

We now give an example which in some sense has constructions very similar to those described above: we add some generic relations to the structure. On the other hand, the effect of this will be opposite. In this construction we use reducts of the random graph [13]. 
Proposition 3.3. There exists a simple $\omega$-categorical theory such that for any finite set of parameters $A$, no non-algebraic type over $A$ extends to a strongly determined type.

Proof. The construction uses ideas from [6]; in fact, Dugald Macpherson pointed out to the author that these ideas can be applied to such an example.

Let $L_{0}=\left\{R_{1}, R_{2}, \ldots, R_{n}, \ldots\right\}$ be a relational language, where each $R_{i}$ has arity $2 i$. The structure $M_{0}$ is built by a Fraissé construction, so we first specify a class $K$ of finite $L_{0}$-structures. In each $C \in K$ each relation $R_{n}$ determines a graph on the set (denoted by $\left(\begin{array}{l}C \\ n\end{array}\right)$ )) of unordered $n$-element subsets of $C$. It is easy to see that $K$ is an amalgamation class: given $A, B_{1}, B_{2} \in K$ with $B_{1} \cap B_{2}=A$, define $C \in K$ as $B_{1} \cup B_{2}$, so that no tuple $\bar{c}_{1} \bar{c}_{2} \in C$ which satisfies $R_{n}$ meets both $B_{2} \backslash B_{1}$ and $B_{1} \backslash B_{2}$. Let $M_{0}$ be the corresponding universal homogeneous structure. Note that $\operatorname{Th}\left(M_{0}\right)$ admits elimination of quantifiers.

Claim A. The theory of $M_{0}$ is supersimple of SU-rank 1.

Let $\phi(\bar{x}, \bar{b}),|\bar{x}|=l$, be a quantifier-free formula and $\left(\bar{b}_{i}: i<\omega\right)$ be an indiscernible sequence of $\operatorname{tp}(\bar{b})$. We may assume that $\phi(\bar{x}, \bar{b})$ implies $\bar{x} \cap \bar{b}=\emptyset$. Then any set $B_{n}=\bigcup\left\{\bar{b}_{i}: i \leq n\right\}$ can be extended by a tuple $c_{1}, \ldots, c_{l}$ satisfying all $\phi\left(\bar{x}, \bar{b}_{i}\right), i \leq n$. Since $M_{0}$ is universal homogeneous, the tuple $\bar{c}$ can be found in $M_{0}$. We now see that any non-algebraic type does not divide over $\emptyset$; thus $M_{0}$ is simple of SU-rank 1 .

Let $M$ be the reduct of $M_{0}$ to the language $L=\left\{T_{1}, \ldots, T_{n}, \ldots\right\}$ of $3 n$-relations (of two-graphs) where a triple of $n$-element sets $C_{1}, C_{2}$ and $C_{3}$ satisfies $T_{n}$ if and only if it contains one or three edges with respect to $R_{n}$. In this case any quadrangle of $n$-element sets has even $T_{n}$-triples. By Claim A the structure $M$ is supersimple. It is easy to see (by genericity) that for all $\bar{a}$ and $A, \operatorname{tp}(\bar{a} / A) \vdash \operatorname{tp}\left(\bar{a} / \operatorname{acl}^{\mathrm{eq}}(A)\right)$ with respect to both $\operatorname{Th}\left(M_{0}\right)$ and $\operatorname{Th}(M)$.

Claim B. Let $C=\left\{c_{1}, \ldots, c_{n}\right\} \subseteq M_{0}$. Let $R_{n}^{\prime}$ be the relation which coincides with $R_{n}$ on all pairs $D, B$ with $C \notin\{D, B\}$ but for any $D \in\left(\begin{array}{c}M \\ n\end{array}\right)$ we have: $(C, D) \in R_{n} \leftrightarrow(C, D) \notin R_{n}^{\prime}$. Then the structure $M_{0}$ is isomorphic to $M_{0}^{\prime}=\left(M, R_{1}, \ldots, R_{n-1}, R_{n}^{\prime}, R_{n+1}, \ldots\right)$ and the structure $M$ is the reduct of $M_{0}^{\prime}$ obtained by the same definition as $M$ is obtained from $M_{0}$.

To prove the claim it suffices to note that any structure from $K$ is embeddable into $M_{0}^{\prime}$ and for every pair $A<A^{\prime}$ from $K$ with $A^{\prime} \cap M_{0}^{\prime}=A$ there exists an $A$-embedding of $A^{\prime}$ into $M_{0}^{\prime}$ (verifying the latter condition we may assume that $C \subseteq A$ ). Both conditions follow from the fact that $M_{0}$ is universal homogeneous. The second statement of the claim is obvious.

Let $\bar{a}=\left(a_{1}, \ldots, a_{n}\right) \subset M$. Let $p(x)$ be a type over $\bar{a}$ which extends to a strongly determined type, and let $a_{0}$ be a realization over $M$ of the corresponding strongly determined type. Since $M_{0}$ is universal homogeneous, 
there exists $b \in M$ realizing $p$ such that $\operatorname{tp}\left(a_{0} b / \bar{a}\right)=\operatorname{tp}\left(b a_{0} / \bar{a}\right)$ with respect to both $M_{0}$ and $M$ (for example one can assume that there is no $R_{l}$-relation between any $l$-sets $C \neq D$ with $\left\{a_{0}, b\right\} \subseteq C \cup D \subseteq \bar{a} \cup\left\{a_{0}, b\right\}, l>0$ ). Since $M_{0}$ is universal homogeneous, there are elements $c, d, d^{\prime} \in M_{0} \backslash \bar{a}$ so that the following conditions hold in $M_{0}$ :

$$
\operatorname{tp}\left(a_{0} b / \bar{a}\right)=\operatorname{tp}(b c / \bar{a})=\operatorname{tp}(b d / \bar{a})=\operatorname{tp}(c d / \bar{a})=\operatorname{tp}\left(c d^{\prime} / \bar{a}\right)
$$

and for every proper subtuple $\bar{a}^{\prime} \subset \bar{a}, \operatorname{tp}\left(a_{0} b / \bar{a}^{\prime}\right)=\operatorname{tp}\left(b d^{\prime} / \bar{a}^{\prime}\right)$. We also assume that $\left(b \bar{a}, d^{\prime} \bar{a}\right) \in R_{n+1}$ if and only if $(b \bar{a}, d \bar{a}) \notin R_{n+1}$, and for any pair $C_{1}, C_{2}$ with $C_{1} \cup C_{2}=d b \bar{a}$, distinct from $b \bar{a}, d \bar{a}$, the corresponding pair $C_{1}^{\prime}$ and $C_{2}^{\prime}$ (obtained by replacing $d$ by $d^{\prime}$ ) satisfies $R_{n+1}$ if and only if $C_{1}, C_{2}$ does.

Let $R_{n+1}^{\prime}$ be obtained from $R_{n+1}$ as in Claim B (by switching) with respect to the (unordered) tuple $d \bar{a}$. Since the structure $M_{0}^{\prime}=\left(M, R_{1}, \ldots, R_{n}\right.$, $\left.R_{n+1}^{\prime}, R_{n+2}, \ldots\right)$ is isomorphic to $M_{0}$, the type of $b d^{\prime}$ over $\bar{a}$ in $M_{0}$ is the same as the type of $b d$ over $\bar{a}$ in $M_{0}^{\prime}$ (by our construction mutually corresponding subtuples from $b d \bar{a}$ and $b d^{\prime} \bar{a}$ satisfy the same relations). Applying the last statement of Claim B we see that the type of $b d$ over $\bar{a}$ in $M$ is the same as the type of $b d^{\prime}$ over $\bar{a}$ in $M$.

Let $q=\operatorname{tp}_{M}(b d / \bar{a})$. Since $a_{0}$ realizes over $M$ the corresponding strongly determined type, one of the following cases holds: (a) for every pair $c^{\prime} c^{\prime \prime} \in M$ realizing $q$ the triple $\left(a_{0} \bar{a}, c^{\prime} \bar{a}, c^{\prime \prime} \bar{a}\right)$ belongs to $T_{n+1}$, or (b) for every pair $c^{\prime} c^{\prime \prime} \in M$ realizing $q$ the triple $\left(a_{0} \bar{a}, c^{\prime} \bar{a}, c^{\prime \prime} \bar{a}\right)$ does not belong to $T_{n+1}$.

In both cases one of the sets $\left\{a_{0} \bar{a}, b \bar{a}, c \bar{a}, d \bar{a}\right\}$ or $\left\{a_{0} \bar{a}, b \bar{a}, c \bar{a}, d^{\prime} \bar{a}\right\}$ has the property that an odd number of triples satisfy $T_{n+1}$. This contradicts the definition of a two-graph.

4. $\mathrm{KP}(\mathrm{L})$-determined types and G-compact types. The results above motivate the following question. Is there a theory admitting (having) strongly determined 1-types and having a strongly determined type which is not G-trivial? By Proposition 1.6(2) such a theory would be a principally new example of a non-G-compact theory, because all known examples have $E_{\mathrm{L}}=E_{\mathrm{KP}}$ on finite tuples or do not have strongly determined types. In this section we formulate further questions of this kind. They are motivated by some remarks concerning possible generalizations of strongly determined types and G-triviality.

4.1. KP-determined and L-determined types. Let $A \subseteq \mathbf{C}$ and $A \subseteq \mathcal{A} \subset$ $\operatorname{bdd}(A)\left(^{5}\right)$. If $q(\bar{y}) \in S(\operatorname{bdd}(\mathcal{A}))$, we say that a type $p(\bar{x}, \bar{y}) \in S(\mathcal{A})$ is a $q$-consistent $\bar{x}$-type if for any sequence $\bar{a}_{1}, \ldots, \bar{a}_{n}$ of realizations of $q$ the set $\bigcup\left\{p\left(\bar{x}, \bar{a}_{i}\right): 1 \leq i \leq n\right\}$ is consistent. A KP-determined type over a set $\mathcal{A}$ is a

$\left(^{5}\right)$ In fact we may assume that $\mathcal{A}$ is an arbitrary set of hyperimaginaries. 
monotonic function $\varrho$ which assigns a $q$-consistent $\bar{x}$-type $\varrho(q)(\bar{x}, \bar{y})$ to every $q(\bar{y}) \in S(\operatorname{bdd}(\mathcal{A}))$.

Suppose that $\varrho$ is a KP-determined type over $\mathcal{A}$. For every $B \subset \mathbf{C}$ define

$$
\varrho_{B}(\bar{x})=\bigcup\{\varrho(q)(\bar{x}, \bar{b}): q \in S(\operatorname{bdd}(\mathcal{A})), \bar{b} \models q, \bar{b} \in B\} .
$$

The definition is a generalization of strongly determined types. The notions are the same if $E_{\mathrm{KP}}^{A}=E_{\mathrm{Sh}}^{A}$. We now see that Theorem 2.2 implies that under $E_{\mathrm{KP}}=E_{\mathrm{L}}$ and admitting strongly determined 1-types, KP-determined types are strongly determined.

To obtain the definition of $L$-determined types we modify the definition of KP-determined types by the requirement that the types $q(\bar{y})$ in that definition are Lascar strong types over $\mathcal{A}$. As above, for an L-determined type $\varrho$ and a set $B$ we define $\varrho_{B}$.

We now say that a theory $T$ admits KP-determined (resp. L-determined) types over $A \subset \mathbf{C}$ if every type of $S(A)$ extends to a KP-determined (resp. L-determined) one. A theory $T$ admits KP-determined (resp. L-determined) types if it admits KP-determined (resp. L-determined) types over every set $A \subset \mathbf{C}$ of parameters. Since a strongly determined type naturally defines a KP-determined type and a KP-determined type naturally defines an Ldetermined type, the class of theories admitting strongly determined types is contained in the class of those admitting KP-determined (or L-determined) types. This suggests that the material from [6] and above can be slightly extended. For example note that the KP- and L-versions of Theorem 2.1 are much easier than the corresponding version for strongly determined types.

Proposition 4.1. Let $T$ be a complete theory such that for any finite $A$ of the basic sort every 1-type over $A$ extends to an L-determined type over $A$. Then for all tuples $\bar{a} \in \mathbf{C}$ and all $n>0$, every $n$-type of $T$ over $\bar{a}$ has an $L$-determined extension over $\bar{a}$. If $E_{\mathrm{L}}^{A}=E_{\mathrm{KP}}^{A}$ on finite tuples (for example $T$ is $\omega$-categorical), then the same statement holds for KP-determined types.

Proof. Let $\varrho$ be an L-determined type over $\bar{a}$.

Claim. For any $\bar{b}, \bar{b}^{\prime}$ and $\bar{c}=\varrho_{\bar{b} \bar{b}^{\prime}}$, if $\left(\bar{b}, \bar{b}^{\prime}\right) \in E_{\mathrm{L}}$ over $\bar{a}$, then $\left(\bar{b}, \bar{b}^{\prime}\right) \in E_{\mathrm{L}}$ over $\bar{a} \bar{c}$.

Proof of Claim. Find $\bar{b}_{0}(=\bar{b}), \bar{b}_{1}, \ldots, \bar{b}_{n}\left(=\bar{b}^{\prime}\right)$ such that every pair $\bar{b}_{i}$, $\bar{b}_{i+1}$ belongs to some infinite $\bar{a}$-indiscernible sequence $I_{i}, i=0, \ldots, n-1$. Then for any realization $\bar{c}_{i} \models \varrho_{\bar{a} I_{i}}$ the tuples $\bar{b}_{i}$ and $\bar{b}_{i+1}$ have the same Lascar strong type over $\bar{a} \bar{c}_{i}$. This implies that if $\bar{c} \models \varrho_{\bar{b}_{0}, \ldots, \bar{b}_{n}}$, then all $\bar{b}_{i}$ have the same Lascar strong type over $\bar{a} \bar{c}$.

Now to finish the proof of the proposition it suffices to repeat the proof of Proposition 2.3 replacing everywhere strong and strongly determined types by Lascar strong and L-determined types respectively. 
Under the assumption $E_{\mathrm{L}}^{A}=E_{\mathrm{KP}}^{A}$ on finite tuples the claim above becomes the corresponding claim for KP-determined types. Therefore it is clear that the proposition holds for KP-determined types.

As in Section 1 we can show that in fact the definitions of KP-determined and L-determined types over $\mathcal{A}$ do not change if we strengthen them by the requirement that $\varrho(q)(\bar{x}, \bar{y})$ is a type over $\operatorname{bdd}(\mathcal{A})$ (resp. is a Lascar strong type over $\mathcal{A}$ ).

Lemma 4.2. Let $A \subset \mathbf{C}$ and $A \subseteq \mathcal{A} \subset \operatorname{bdd}(A)$. Let $\varrho(\bar{x})$ be a $K P$ determined (resp. L-determined) type over $\mathcal{A}$. Then there exists a unique KP-determined type $\varrho^{\prime}(\bar{x})$ over $\operatorname{bdd}(A)$ (resp. L-determined type over the set of classes of all bounded $\operatorname{Aut}(\mathbf{C} / A)$-invariant equivalence relations) such that for any KP-strong (resp. Lascar strong type) $q, \varrho^{\prime}(q) \vdash \varrho(q)$.

Proof. We start with the case when $\varrho$ is KP-determined. Let $M$ be very $K P$-rich over $A$ : for all $n \in \omega$ and all $\bar{m} \in M, M$ realizes all $n$-types from $S(\operatorname{bdd}(A \bar{m}))$. Let $\bar{c} \models \varrho_{M}$. For $q \in S(\operatorname{bdd}(A))$ and $\bar{b} \models q, \bar{b} \in M$, define $\varrho^{\prime}(q):=\operatorname{tp}(\bar{c} \bar{b} / \operatorname{bdd}(A))$.

To see that the definition is correct, repeat the corresponding proof of Lemma 1.1 replacing everywhere finite equivalence relations by bounded type-definable equivalence relations, and strong types by KP-strong types.

In the case when $\varrho$ is L-determined let $M$ be very $L$-rich over $A$ : for all $n \in \omega$ and all $\bar{m} \in M, M$ realizes all Lascar strong $n$-types over $A \bar{m}$. Let $\bar{c} \models \varrho_{M}$. For a Lascar strong type $q$ over $A$ and $\bar{b} \models q, \bar{b} \in M$, define $\varrho^{\prime}(q)$ to be the Lascar strong type of $\bar{c} \bar{b}$ over $A$.

To see that the definition is correct take any $\bar{b}, \bar{b}^{\prime} \in M$ of the same Lascar strong type over $A$. There are $\bar{b}_{0}(=\bar{b}), \bar{b}_{1}, \ldots, \bar{b}_{n}\left(=\bar{b}^{\prime}\right)$ such that every pair $\bar{b}_{i}, \bar{b}_{i+1}$ belongs to some infinite $A$-indiscernible sequence $I_{i}, i=0, \ldots, n-1$. We may assume that these sequences consist of tuples from $M$. Since $\bar{c}=$ $\varrho_{I_{i} A}$, the tuples $\bar{b}_{i}$ and $\bar{b}_{i+1}$ have the same Lascar strong type over $A \bar{c}$. This implies that all $\bar{b}_{i}$ have the same Lascar strong type over $A \bar{c}$. In particular $\bar{c} \bar{b}$ and $\bar{c} \bar{b}^{\prime}$ have the same Lascar strong type over $A$.

Repeating the proof of Lemma 1.2 we obtain

Lemma 4.3. Let $T$ admit KP-determined (resp. L-determined) n-types over a set $A$. Then every KP-strong (resp. L-strong) $n$-type over A extends to a KP-determined (resp. L-determined) type.

The compactness argument from the proof of Lemma 2.1 from [6] can be easily applied to the following lemma.

Lemma 4.4. Suppose that $M$ is very KP-rich (resp. L-rich) over A. Let $p(\bar{x}) \in S(A)$. Then the following are equivalent:

(i) $p$ extends to a KP-determined (resp. an L-determined) type over A; 
(ii) for every finite set $\Gamma$ of finite partial KP-strong (L-strong) elementary maps $M \rightarrow M$ there exists $\bar{c} \in p(M)$ such that all the maps in $\Gamma$ are elementary over $\bar{c} A$.

We now see by inspection that Proposition 3.1 also holds for KP-determined (resp. L-determined) types.

The proof of Proposition 1.4 also works for the following statement: If a theory $T$ admits KP-determined types, then $T$ is G-compact.

4.2. G-compact strongly determined types. Let $\varrho$ be a KP-determined type over $\mathcal{A}$. Let $M$ be sufficiently saturated, $\mathcal{A} \subseteq M^{\text {eq }}, \bar{b} \models \varrho_{M}$ and $e$ be a bounded $\bar{b} \mathcal{A}$-type-definable equivalence relation. Let $\widetilde{e}$ be the equivalence relation defined on the appropriate $\mathbf{C}^{l}$ by the condition that $\left(\bar{a}, \bar{a}^{\prime}\right) \in \widetilde{e}$ if and only if the corresponding $e$-classes of $\bar{a}$ and $\bar{a}^{\prime}$ are in the same orbit with respect to $\operatorname{Aut}(\mathbf{C} / \bar{b} M)$.

The group $\operatorname{Aut}(\mathbf{C} /\{M\} \operatorname{bdd}(\mathcal{A}) \bar{b})$ has a natural action on $\widetilde{e}$-classes. By the choice of $\bar{b}$ any automorphism from $\operatorname{Aut}_{\mathrm{KP}}(M / \mathcal{A})$ extends to an element of $\operatorname{Aut}(\mathbf{C} / \bar{b}\{M\} \operatorname{bdd}(\mathcal{A})$ ) (fixing $M$ setwise). We have obtained the exact sequence

$$
0 \rightarrow \operatorname{Aut}(\mathbf{C} / \bar{b} M) \rightarrow \operatorname{Aut}(\mathbf{C} / \bar{b}\{M\} \operatorname{bdd}(\mathcal{A})) \rightarrow \operatorname{Aut} \operatorname{KP}(M / \mathcal{A}) \rightarrow 0
$$

where the kernel $\operatorname{Aut}(\mathbf{C} / \bar{b} M)$ preserves all $\widetilde{e}$-classes. Hence we deduce that $\operatorname{Aut}(\mathbf{C} / \bar{b}\{M\} \operatorname{bdd}(\mathcal{A}))$ induces an action of $\operatorname{Aut}_{\mathrm{KP}}(M / \mathcal{A})$ on the set of $\widetilde{e}-$ classes.

Since $e$ is a bounded equivalence relation the pointwise stabilizer of the set of $\widetilde{e}$-classes is a normal subgroup $H_{e} \triangleleft \operatorname{Aut}_{\mathrm{KP}}(M / \mathcal{A})$ of bounded index.

Definition 4.5. We say that $\varrho$ is $G$-compact if for any sufficiently homogeneous structure $M$ realizing all KP-strong types over $\mathcal{A}$ and any bounded equivalence relation $e$ as above, there is no $\operatorname{Aut}_{\mathrm{KP}}(M / \mathcal{A})$-invariant equivalence relation $E$ refining $E_{\mathrm{KP}}^{\mathcal{A}, l}$ on the appropriate $M^{l}$, such that the corresponding group $H_{e}$ preserves all $E$-classes.

For simplicity let $\mathcal{A}=A \subset \mathbf{C}$. It is worth noting that if an equivalence relation $E$ witnesses non-G-compactness then each $E_{\mathrm{KP}}^{A, l}$-class on $M^{l}$ consists of $\leq\left|\operatorname{Aut}_{\mathrm{KP}}(M / A): H_{e}\right|$ classes of $E$. Since the intersection of a bounded number of subgroups of $\operatorname{Aut}(M / A)$ of bounded index also has bounded index, we see that in this case $\operatorname{Th}(M, a)_{a \in A}$ is not G-compact (the intersection of all conjugates of $E$ contradicts G-compactness). Since we do not know too many examples of non-G-compact theories, the case of non-G-compact KPdetermined types looks slightly artificial. Nevertheless we conjecture that such KP-determined types exist.

REMARK. It is worth noting that replacing Aut $_{\mathrm{KP}}$ and $e$ above by $\mathrm{Aut}_{\mathrm{L}}$ and a bounded equivalence relation invariant over $\bar{b} A$ respectively we ob- 
tain another version of G-compactness. This version is satisfied by any Ldetermined type because $E_{\mathrm{L}}^{A, l}$ is the finest bounded equivalence relation.

Now let $\varrho$ be a strongly determined type over $A \subset$ C. Let $M$ be sufficiently saturated and homogeneous, $\bar{b} \models \varrho_{M}$ and $e$ be a finite equivalence relation definable over $A \bar{b}$. Let $\widetilde{e}$ be the equivalence relation defined on the appropriate $\mathbf{C}^{l}$ by the condition that $\left(\bar{a}, \bar{a}^{\prime}\right) \in \widetilde{e}$ if and only if the corresponding $e$-classes of $\bar{a}$ and $\bar{a}^{\prime}$ are in the same orbit with respect to $\operatorname{Aut}(\mathbf{C} / \bar{b} M)$. We know that the pointwise stabilizer of the set of $\widetilde{e}$-classes is a normal subgroup $H_{e} \triangleleft \operatorname{Aut}\left(M / \operatorname{acl}^{\mathrm{eq}}(A)\right)$ of finite index.

We say that $\varrho$ is $G$-finite if the intersection of all subgroups of $\operatorname{Aut}_{\mathrm{Sh}}(M / A)$ of the form $H_{e}$ for finite equivalence relations $e$ as above is of finite index in $\operatorname{Aut}_{\mathrm{Sh}}(M / A)$. The following question looks interesting. Is a G-finite strongly determined type G-trivial? We know from Section 1.2 that the theory of the corresponding counterexample must be non-G-compact. Moreover, it looks likely that a G-finite strongly determined type naturally defines a G-compact KP-determined type (this may be connected with the question finishing Section 1).

\section{References}

[1] E. Casanovas, D. Lascar, A. Pillay and M. Ziegler, Galois groups of first order theories, J. Math. Logic 1 (2001), 305-319.

[2] Z. Chatzidakis and E. Hrushovski, Model theory of difference fields, Trans. Amer. Math. Soc. 351 (1999), 2997-3071.

[3] Z. Chatzidakis and A. Pillay, Generic structures and simple theories, Ann. Pure Appl. Logic 95 (1998), 71-92.

[4] D. Haskell, E. Hrushovski and H. D. Macpherson, Definable sets in algebraically closed valued fields. Part I: elimination of imaginaries, J. Reine Angew. Math., to appear.

[5] - - - - Definable sets in algebraically closed valued fields. Part II: stable domination and independence, submitted.

[6] A. Ivanov and D. Macpherson, Strongly determined types, Ann. Pure Appl. Logic 99 (1999), 197-230.

[7] B. Kim, A note on Lascar strong types in simple theories, J. Symbolic Logic 63 (1998), 926-936.

[8] K. Krupiński and L. Newelski, On bounded type definable equivalence relations, Notre Dame J. Formal Logic 43 (2002), 231-242.

[9] D. Lascar, On the category of models of a complete theory, J. Symbolic Logic 47 (1982), 249-266.

[10] D. Lascar and A. Pillay, Hyperimaginaries and automorphism groups, ibid. 66 (2001), 127-143.

[11] J. Mycielski, Some properties of connected compact groups, Colloq. Math. 5 (1958), $162-166$.

[12] L. Newelski, The diameter of a Lascar strong type, Fund. Math. 176 (2003), 157-170.

[13] S. Thomas, Reducts of the random graph, J. Symbolic Logic 56 (1991), 176-181. 
[14] M. Ziegler, Introduction to the Lascar group, in: Tits Buildings and the Model Theory of Groups, K. Tent (ed.), London Math. Soc. Lecture Note Ser. 291, Cambridge Univ. Press, 2002, 279-298.

Institute of Mathematics

Wrocław University

Pl. Grunwaldzki 2/4

50-384 Wrocław, Poland

E-mail: ivanov@math.uni.wroc.pl

Received 12 May 2005;

in revised form 28 March 2006 